\title{
BMJ Open Swiss Frailty Network and Repository: protocol of a Swiss Personalized Health Network's driver project observational study
}

\author{
Michael Gagesch (D) ,1,2 Karin Edler, ${ }^{3}$ Patricia O Chocano-Bedoya, ${ }^{2,4}$ \\ Lauren A Abderhalden, ${ }^{2}$ Laurence Seematter-Bagnoud, ${ }^{5,6}$ Tobias Meyer, ${ }^{7}$ \\ Dominic Bertschi, ${ }^{8}$ Dina Zekry, ${ }^{9}$ Christophe J Büla (D) , ${ }^{6}$ Gabriel Gold, ${ }^{10}$ \\ Reto W Kressig, ${ }^{7,11}$ Andreas E Stuck, ${ }^{8}$ Heike A Bischoff-Ferrari (1) ${ }^{1,2}$
}

To cite: Gagesch M, Edler K, Chocano-Bedoya PO, et al. Swiss Frailty Network and Repository: protocol of a Swiss Personalized Health Network's driver project observational study. BMJ Open 2021;11:e047429. doi:10.1136/ bmjopen-2020-047429

- Prepublication history for this paper is available online. To view these files, please visit the journal online (http://dx.doi org/10.1136/bmjopen-2020047429).

Received 28 November 2020 Accepted 30 June 2021

Check for updates

(c) Author(s) (or their employer(s)) 2021. Re-use permitted under CC BY-NC. No commercial re-use. See rights and permissions. Published by BMJ.

For numbered affiliations see end of article.

Correspondence to Dr Michael Gagesch michael.gagesch@usz.ch

\section{ABSTRACT}

Introduction Early identification of frailty by clinical instruments or accumulation of deficit indexes can contribute to improve healthcare for older adults, including the prevention of negative outcomes in acute care. However, conflicting evidence exists on how to best capture frailty in this setting. Simultaneously, the increasing utilisation of electronic health records (EHRs) opens up new possibilities for research and patient care, including frailty.

Methods and analysis The Swiss Frailty Network and Repository (SFNR) primarily aims to develop an electronic Frailty Index (eFI) from routinely available EHR data in order to investigate its predictive value against length of stay and in-hospital mortality as two important clinical outcomes in a study sample of 1000-1500 hospital patients aged 65 years and older. In addition, we will examine the correlation between the eFI and a test-based clinical Frailty Instrument to compare both concepts in Swiss older adults in acute care settings. As a Swiss Personalized Health Network (SPHN) driver project, our study will report on the characteristics and usability of the first nationwide eFI in Switzerland connecting all five Swiss University Hospitals' Geriatric Departments with a representative sample of patients aged 65 years and older admitted to acute care.

Ethics and dissemination The study protocol was approved by the competent ethics committee of the Canton of Zurich (BASEC-ID 2019-00445). All acquired data will be handled according to SPHN's ethical framework for responsible data processing in personalised health research. Analyses will be performed within the secure BioMedIT environment, a national infrastructure to enable secure biomedical data processing, an integral part of SPHN.

Trial registration number NCT04516642.

\section{INTRODUCTION}

With the ongoing demographical transformation, ageing societies convey an important challenge to present healthcare systems due to the growing number of older adults living

\section{Strengths and limitations of this study}

- This large multicentre study, recruiting 1000-1500 individuals will establish a new harmonised electronic Frailty Index (eFI) from routinely collected electronic patient data at all five Swiss academic geriatric centres.

- The new eFI has the potential to predict two very important adverse outcomes in acute care, length of stay and in-hospital mortality.

- Furthermore, these new data will be used to investigate the correlation between the eFI and a standardised clinical Frailty Instrument.

- Our study is not intended to establish long-term outcomes in participants identified as frail versus their robust counterparts.

- No report on potential interventions for participants identified as frail or at-risk of becoming frail is included at this stage of the project, however we aim to lay groundwork for a future implementation of frailty screening in clinical care.

with accumulating deficits, multimorbidity and frailty. ${ }^{1}$ At the same time, healthcare informatics with its expanding amount of routinely collected, electronic patient data comprises a huge potential for the exploitation of data for research purposes and future developments in personalised medicine. In order to avoid age discrimination, this should also include the utilisation of electronic patient data in the interest of older adults.

Over the last two decades, frailty was characterised as an age-associated disproportionate decline in physiological reserves leading to increased vulnerability to external stressors, ${ }^{2}$ and shown to be an important predictor of negative health outcomes in older adults. ${ }^{3}$ Nonetheless, frailty is still underdiagnosed in acute care although frail older adults have more frequent and longer hospital stays, are 
re-hospitalised more often and eventually die earlier than their non-frail counterparts. ${ }^{34}$

The British Geriatrics Society issued a recommendation for routine frailty screening in geriatric outpatients in order to timely assess the risk of frailty on the health of older adults. ${ }^{5}$ Moreover, frailty is becoming more and more recognised as a useful concept for risk stratification in various medical specialties, from oncology to heart surgery. ${ }^{6}$ However, as the field is evolving, there has been no agreement either on the ideal conceptualisation of frailty or on a single best screening instrument over the past decades. ${ }^{7-10}$ This happened to be a major roadblock for the broader implementation of the frailty concept into patient care. ${ }^{11}$ At the same time, assessing frailty systematically in clinical care might open up a window of opportunity for both improving patient care for older adults and accelerating research efforts for a better understanding of the underlying pathophysiology of frailty as a state or condition (ie, by a Frailty Index (FI) approach) and as a syndrome (ie, the frailty phenotype)..$^{10}$

Today, among the highly cited frailty conceptualisations, the frailty phenotype by Fried $e t a l^{12}$ and the deficit accumulation concept (ie, FI) by Mitnitski $e t a l^{13}$ stand out as the two most extensively investigated approaches of frailty. ${ }^{14}$ As this two approaches measure different concepts of frailty (ie, a clinical syndrome vs a multisystem decline based index), their comparability may be limited. In addition, it should be taken into account that the phenotype usually requires clinical measurements, whereas a FI can be generated from available patient data collected during routine clinical practice. Therefore, deriving a FI from electronic health records (EHRs) data that are routinely collected, has the potential to expedite the routine identification of frail patients in acute hospital care in various medical specialties, as no additional resources are needed. ${ }^{15}$ This approach has been recently demonstrated by Cesari et al investigating a FI in an Italian cohort of hospitalised patients. ${ }^{16}$ With regard to the investigation of significant clinical endpoints including in-hospital mortality and length of stay (LOS) by an electronic Frailty Index (eFI), which has not been undertaken in hospitalised older adults in Switzerland so far, a comparative view on both frailty concepts contains the opportunity to provide important additional information.

The Swiss Frailty Network and Repository (SFNR) aims to establish a nationwide harmonised eFI consisting of 55 variables from routinely collected EHR data in patients aged 65 years and older at all five Swiss University Hospitals in order to investigate its predictive abilities in regard to LOS and in-hospital mortality. A secondary validation aim investigates the correlation of the eFI as a screening tool against the detection of frailty by a harmonised clinical Frailty Instrument (cFI) based on the Fried phenotype concept, in a subset of patients aged 65 years and older from acute geriatric care at all five Swiss University Hospitals' geriatric centres. In order to take into account the importance of cognitive impairment with regard to frailty, we have added a short cognitive test as an additional component to the cFI. ${ }^{17} 18$ We will investigate the predictive abilities of both, the eFI and the cFI regarding two important outcomes in acute hospital care, LOS and in-hospital mortality. The development of a frailty data repository will in addition serve as a basic personalised health research infrastructure for future studies in older adults across all partner institutions.

The utilisation of routinely collected, electronic patient data is a major focus area in healthcare, and of growing interest in many acute care settings, including geriatric medicine. Frailty is highly prevalent in older patients and appears as a major driver of multiple negative outcomes in this population. Establishing a harmonised eFI from routinely collected EHR data is therefore a timely effort that will likely contribute to the improvement of care for older adult patients by early identifying those at increased risk for adverse outcomes.

The main deliverable of the SFNR will be to establish a nationwide eFI derived from routinely collected electronic patient data for older adults in Switzerland curated within the Swiss Personalized Health Network (SPHN) BioMedIT ecosystem. We aim to demonstrate the eFI's predictive ability for LOS and in-hospital mortality and investigate the comparative performance of the eFI in the detection of frailty against our cFI in a subset of patients admitted to acute geriatric care.

Our proposition of a systematic clinical evaluation of frailty using the cFI as a clinical research reference standard in all enrolled patients admitted to acute geriatric care at all five Swiss Academic geriatric centres is a secondary outcome of our collaboration that may lead to a more unified approach to the measurement of the frailty phenotype on the national level. Therefore, establishing the SFNR will likely advance both, the field of geriatric medicine and research in Switzerland. Incorporating frailty as a criterion in acute care will allow a systematic and personalised pre-therapeutic stratification of patients according to each patient's profile. This individualised approach will enhance the definition of person-based potential harms and benefits of interventions in various medical disciplines, ranging from emergency medicine and orthogeriatric units to cardiovascular surgery and comprehensive cancer care. We expect first results to be ready for scientific publication by mid 2022 .

\section{METHODS AND ANALYSIS}

The SFRN is a joint effort by all five Swiss Academic Geriatric Departments (Universities of Basel, Bern, Geneva, Lausanne and Zurich and adjacent University Hospitals) that is funded by the SPHN (grant no. 2017DRI02), an initiative of the Swiss Federal Government, namely the State Secretariat for Education, Research and Innovation and the Federal Office of Public Health. ${ }^{19}$

The SFNR has five primary aims:

1. Reaching a consensus on a nationwide research reference standard to assess frailty clinically in geriatric 
Table 1 Components of the SFNR clinical Frailty Instrument

\begin{tabular}{|c|c|c|}
\hline Domain (item) & Operationalisation (test-based) & Cut-point (threshold) \\
\hline Shrinking (weight loss) & $\begin{array}{l}\text { Unintentional weight loss or loss of appetite; report of lose } \\
\text { clothing, weight loss documented in patient chart }\end{array}$ & $\begin{array}{l}\text { Any reported weight loss or loss of } \\
\text { appetite or lose clothing or } \\
>5 \% \text { last } 6 \text { months (from EHR) }\end{array}$ \\
\hline Fatigue & $\begin{array}{l}\text { Self-reported exhaustion measured by Geriatric } \\
\text { Depression Scale (GDS) } 4 \text { item }\end{array}$ & $\geq 2$ Points on GDS- $4^{3132}$ \\
\hline Slowness & $\begin{array}{l}\text { Slow gait speed on standardised } 4 \mathrm{~m} \text { measurement from a } \\
\text { standing start (best of two consecutive measurements) }\end{array}$ & Gait speed below $0.8 \mathrm{~m} / \mathrm{s}$ \\
\hline Weakness & $\begin{array}{l}\text { Low hand grip strength measured by the Martin } \\
\text { Vigorimeter (in Kilopascal), best of three consecutive trials } \\
\text { at the dominant hand at time of assessment }\end{array}$ & $\begin{array}{l}\text { Below the median of lowest } 20 \% \text { (by } \\
\text { gender and age }<75 \text { and } \geq 75 \text { years) } \\
\text { compared with a sample of generally } \\
\text { healthy Swiss older adults (from the DO- } \\
\text { HEALTH study }{ }^{33} 3435\end{array}$ \\
\hline Cognition & Three item recall and clock drawing test (CDT) & $\begin{array}{l}\text { Any error in recall or CDT indicates } \\
\text { cognitive disturbance }\end{array}$ \\
\hline
\end{tabular}

EHR, electronic health record; SFNR, Swiss Frailty Network and Repository.

patients at all five partner sites (definition of cFI, goal 1), see table 1 .

2. Reaching a consensus on the candidate variables aggregating to a harmonised eFI from regularly collected electronic patient data extracted from the local clinical information systems (CISs) at all five sites (goal 2), table 2.

3. Setting up of a frailty data hub for the collection, organisation and maintenance of coded data from all five centres including both, the cFI from patients in acute care seen by the geriatric teams at each site (related to goal 1) and the harmonised eFI (related to goal 2) from all patients aged 65 years and older at the partnering Swiss University Hospitals.

4. Investigating the correlation of the eFI as a screening tool against the cFI as a clinical criterion standard within the pooled data set from all five geriatric centres (association study).

5. Investigating whether the prognostic abilities differ between the cFI and the eFI with regard to the prediction of LOS and in-hospital mortality in acute geriatric care (correlation study).

\section{Sample size calculation}

For our study, the estimated total sample size of 1000-1500 patients within a 12-month planned period was based on the two primary endpoints, hospital LOS and in-hospital mortality. In a prior study, Hope et al found a median (IQR) LOS in the hospital for non-frail and frail individuals to be 13 (IQR 8-23) days and 17 (IQR 10-30) days, respectively. ${ }^{20}$ Assuming symmetry, this translates to a mean $(\mathrm{SD})$ of $13(\mathrm{SD}=(23-8) / 1.35=11.1)$ days for nonfrail individuals and 17 (14.8) days for frail individuals. ${ }^{21}$ Another study investigating older adult medical inpatients found a range in LOS between 4.2 and 7.8 along a
FI score based on a comprehensive geriatric assessment. ${ }^{22}$ In addition, a systematic review and meta-analysis of nine observational studies investigating outcomes in general surgery reported a mean LOS of 9.6 days (95\% CI: 6.2 to 12.9) in frail and 6.4days (4.9 to 7.9) in non-frail patients. ${ }^{23}$ Conservatively assuming $20 \%$ of older adults in acute care are frail (expected range from literature $20 \%-50 \%)^{24}$ and assuming a difference in hospital LOS of 4 days, a total of 418 persons (92 frail, 326 non-frail) would be needed to achieve $80 \%$ power at the 0.05 alpha level. Using a more conservative estimate of detecting a difference in hospital LOS of 2 days, 1655 (364 frail, 1291 non-frail) individuals would be needed to achieve $80 \%$ power at the 0.05 alpha level. Vermeiren et al conducted a meta-analysis of 24 prospective studies comprising over 150000 individuals and found frailty to increase the likelihood of mortality more than twofold (OR 2.34 (1.77 to 3.09$)) .{ }^{3}$ We assume $20 \%$ of individuals are frail, and leave room for a greater degree of uncertainty (wider CI, CI 1.42 to 3.91) since this is a single study as opposed to a large meta-analysis comprising many individuals. For a mortality rate of $5 \%$ in non-frail individuals, a total of 1077 persons (237 frail, 840 non-frail) would be required to detect an $\mathrm{OR}=2.34$ at the 0.05 confidence level.

In summary, we consider a sample size of approximately 200-300 patients enrolled at each of the five partnering sites over the planned 12-month period sufficient to answer our research questions. However, the number of recruited participants at each site might not be equally distributed and differ largely due to the local environments and in-patient capacities. Of note, the primary analysis will be performed on the total sample of 1000 1500 patients. 
Table 2 Variables and coding of the SFNR electronic Frailty Index (eFI)

\begin{tabular}{|c|c|c|c|}
\hline System & Source & Variables (no.) & Cut-point/coding \\
\hline $\begin{array}{l}\text { Functional } \\
\text { impairments }\end{array}$ & $\begin{array}{l}\text { Electronic } \\
\text { health records } \\
\text { (EHRs): } \\
\text { electronic } \\
\text { nursing charts } \\
\text { (NANDA) }\end{array}$ & $\begin{array}{l}\text { 1. Chronic constipation } \\
\text { 2. Feeling tired } \\
\text { 3. Problems with falling/staying asleep } \\
\text { 4. Problems with sleep-awake cycle } \\
\text { 5. Urinary incontinence } \\
\text { 6. Help getting on/off bed } \\
\text { 7. Help going to the toilet } \\
\text { 8. Help walking } \\
\text { 9. History of falls } \\
\text { 10. Inability to walk stairs } \\
\text { 11. Irregular gait pattern } \\
\text { 12. Patient using walking equipment/aid } \\
\text { 13. Problems getting dressed } \\
\text { 14. Problems with bathing } \\
\text { 15. Clouding or delirium } \\
\text { 16. Food intake }\end{array}$ & $\begin{array}{l}\text { Yes }=1, \text { no }=0 \\
\text { impaired }=1, \text { normal }=0\end{array}$ \\
\hline Vital signs & EHRs & $\begin{array}{l}\text { 47. Body temperature } \\
\text { 48. Diastolic blood pressure } \\
\text { 49. Heart rate (pulse) } \\
\text { 50. Systolic blood pressure } \\
\text { 51. Oxygen saturation }\left(\mathrm{SpO}_{2}\right) \\
\text { 52. Patient requires supplemental oxygen }\end{array}$ & $\begin{array}{l}<36,3^{\circ} \mathrm{C}=1, \geq 36,3^{\circ} \mathrm{C}=0 \\
>90 \mathrm{mmHg}=1, \leq 90 \mathrm{mmHg}=0 \\
<60 \text { or }>99 \mathrm{BPM}=1, \text { other }=0 \\
>140 \mathrm{mmHg}=1, \leq 140 \mathrm{mmHg}=0 \\
<90 \%=1, \geq 90 \%=0 \\
\text { yes }=1, \mathrm{no}=0\end{array}$ \\
\hline Other & EHRs & $\begin{array}{l}\text { 53. Age } \\
\text { 54. Body mass index } \\
\text { 55. Patient reports being in pain }\end{array}$ & $\begin{array}{l}>80=1, \leq 80=0 \\
<18.5 \text { or } \geq 30=1 ;>25 \text { and }<30=0.5, \text { other }=0 \\
\text { yes }=1, \text { no }=0\end{array}$ \\
\hline
\end{tabular}

SFNR, Swiss Frailty Network and Repository. 


\section{Data collection}

The data collection for the components of the cFI will take place within the first 4 days on admission to acute geriatric care at all partner sites by certified examiners following a standardised protocol. For calculating the eFI, only variables available from within 4 days on admission will be retrieved from the EHR and included to the dataset.

\section{Statistical analysis}

In regard to the eFI's variables, each will be scored as either ' 1 ', that is, presence of the deficit or ' 0 ', that is, absence of the deficit, except for body mass index $(<18.5$ or $\geq 30=$ $1,>25$ and $<30=0.5$, other $=0$ ), see table 2 for full list of variables. We will use validated cut-points regarding the classification of the degree of robustness or frailty from prior literature. ${ }^{25} 26$ We will additionally test, whether eFI scores differ between the classification of frail/prefrail and robust by the cFI in our subsample from acute geriatric care. To evaluate the ability of the eFI screening tool to correctly classify each patient as frail, pre-frail or non-frail in regard to the phenotypic approach, we will calculate the sensitivity, specificity, as well as positive and negative predictive values of the eFI (pre-defined cut-offs and tertiles) against the $\mathrm{cFI}{ }^{27}$ Each potential threshold will be applied to the continuous total sum scores of the eFI to classify frail vs non-frail participants. The resulting true positive rate (sensitivity) and false positive rate (1specificity) will be determined using the cFI as the reference. A receiver operating characteristic (ROC) curve will be constructed for all possible thresholds of the eFI. Discriminative ability will be estimated based on the area under the ROC curve and associated C statistics.

Hospital LOS in frail and non-frail individuals (classified by eFI and cFI) will be summarised using mean, median, SD, IQR, minimum and maximum. Differences in hospital LOS between frail and non-frail individuals will be tested using a two-sided independent $t$ test, or Mann-Whitney $\mathrm{U}$ test if the data is skewed, at the 0.05 level. In-hospital mortality rates will be calculated for the overall sample as well as for frail and non-frail subgroups. Logistic regression will be used to quantify the association of frail (vs non-frail) on in-hospital mortality.

\section{Progress to date}

In the first year of the project, consensus was reached among the project partners regarding the composition and scoring of the $\mathrm{cFI}$. At the same time, the 55 variables summarised in the eFI were defined and harmonised. In the second year, the local requirements for the provision of the data to be collected were analysed and the required IT infrastructure for secure data processing and delivery was set up. At the same time, the project-related data infrastructure within the BioMedIT network was defined and made available by SPHN. Enrolment of first participants into the study began in June 2020.

\section{Patient and public involvement}

Patients and the general public were not involved in the design, recruitment and implementation of our study. Participants will be informed regarding the detailed results of our study only on request. However, the results will be disseminated to the public according to the SPHN's dissemination policy and by published articles.

\section{ETHICS AND DISSEMINATION}

For the association study, with regard to the eFI, we will use data from consecutive patients aged 65 years and older admitted to acute care on various departments of the partnering university hospitals from a determined starting date and with available written informed consent for further use of routine clinical data. For the correlation study, we will use data from all patients aged 65 years and older recruited from acute geriatric care units who agreed to participate in the study by informed consent.

All ethics committees of the involved partner sites, chaired by the ethics committee of the Canton of Zurich have approved our study (swissethics BASEC-ID 2019-00445).

\section{SPHN IT ecosystem}

Our project's hosting initiative, the SPHN, is currently developing a nationwide healthcare data ecosystem in Switzerland to work towards interoperability of data from local information systems, for example, clinical data management systems in enabling an effective exchange of patient data (eg, disease phenotypes) for research with the ultimate goal of advancing personalised medicine. ${ }^{28} 29$ Our project will support and build on this effort as a driver project. In a first step, the agreed set of eFI variables was submitted to the SPHN Clinical Semantic Interoperability Working Group, which has integrated the variables in a Swiss wide core dataset and is defining for each variable in which format they shall be shared and which additional (meta-) data are needed for optimal interoperability. The data of the test-based cFI will be collected in a standardised and centralised electronic Case Report Form in REDCap (Vanderbilt University, Nashville, Tennessee, USA) or in the CIS (EHR).

Next, our collected data (eFI and cFI) will be locally pre-processed by the clinical data warehouse teams. In particular, patient IDs will be mapped and de-identified before sharing to respect data privacy regulations. ${ }^{19}$ Additionally, a standardised format for data transfer defined by a Data Coordination Center (DCC) will be used in order to allow interoperability. We will used the novel Swiss BioMedIT-Node secure data infrastructure currently under development by the Swiss Institute of Bioinformatics and managed by the Personalized Health Informatics Group and coordinated by the DCC as part of SPHN. ${ }^{30}$ The de-identified data will be encrypted with a secure, standard mechanism (GPG, GNU Privacy Guard) and sent via secure transfer to BioMedIT. 
On BioMedIT, the analysis of the data will take place using state of the art software and tools thereby ensuring highest security levels for access to data, processing and sharing. FAIR data principles (findability, accessibility, interoperability, reusability) will be respected and ensured throughout the project in accordance with SPHN strategy.

\section{Author affiliations}

${ }^{1}$ Department of Geriatrics, University Hospital Zurich, Zurich, Switzerland

${ }^{2}$ Centre on Aging and Mobility, University Hospital Zurich and University of Zurich, Zurich, Switzerland

${ }^{3}$ Research Data Service Center, Clinical Trails Center, University Hospital Zurich, Zurich, Switzerland

${ }^{4}$ Institute of Primary Health Care (BIHAM), University of Bern, Bern, Switzerland ${ }^{5}$ Department of Epidemiology and Health Systems, Center for Primary Care and Public Health, Lausanne, Switzerland

${ }^{6}$ Service of Geriatric Medicine and Geriatric Rehabilitation, Lausanne University Hospital, Lausanne, Switzerland

${ }^{7}$ Universitäre Altersmedizin FELIX PLATTER, Basel, Switzerland

${ }^{8}$ Department of Geriatrics, Inselspital, Bern University Hospital, University of Bern, 3010 Bern, Switzerland

${ }^{9}$ Division of Geriatrics, Department of Internal Medicine, Rehabilitation and Geriatrics, Geneva University Hospitals, Geneva, Switzerland

${ }^{10}$ Department of Rehabilitation and Geriatrics, University Hospitals Geneva, Geneva, Switzerland

${ }^{11}$ Universität Basel, Basel, Switzerland

Contributors MG and KE prepared the first draft of the manuscript. POC-B and LAA provided the power analysis and wrote the section on statistical analysis. LS-B, TM, DB, DZ, CJB, GG, RWK and AES have read and edited the paper for intellectual content and contributed significantly to the manuscript. HAB-F was a major contributor in writing the final manuscript. MG prepared the final editing before submission. All authors read and approved the final manuscript.

Funding This project has been funded as a driver project by the Swiss Personalised Health Network (grant number 2017DRI02) with the participating University Hospitals in-kind contributions of the same amount (matching funds, grant number N/A).

Disclaimer The funding source was not involved in study design, and will not be involved in the collection, analysis or interpretation of data.

\section{Competing interests None declared.}

Patient and public involvement Patients and/or the public were not involved in the design, or conduct, or reporting, or dissemination plans of this research.

Patient consent for publication Not required.

Provenance and peer review Not commissioned; externally peer reviewed.

Open access This is an open access article distributed in accordance with the Creative Commons Attribution Non Commercial (CC BY-NC 4.0) license, which permits others to distribute, remix, adapt, build upon this work non-commercially, and license their derivative works on different terms, provided the original work is properly cited, appropriate credit is given, any changes made indicated, and the use is non-commercial. See: http://creativecommons.org/licenses/by-nc/4.0/.

ORCID iDs

Michael Gagesch http://orcid.org/0000-0003-3089-5768

Christophe J Büla http://orcid.org/0000-0002-7501-3442

Heike A Bischoff-Ferrari http://orcid.org/0000-0002-4554-658X

\section{REFERENCES}

1 Newman AB. Comorbidity and multimorbidity. In: Newman A, Cauley J, eds. The epidemiology of aging. Dordrecht: Springer, 2012.

2 Clegg A, Young J, lliffe S, et al. Frailty in elderly people. Lancet 2013;381:752-62.

3 Vermeiren S, Vella-Azzopardi R, Beckwée D, et al. Frailty and the prediction of negative health outcomes: a meta-analysis. J Am Med Dir Assoc 2016;17:1163.e1-17.
4 Lin H-S, Watts JN, Peel NM, et al. Frailty and post-operative outcomes in older surgical patients: a systematic review. BMC Geriatr 2016;16:157.

5 Turner G, Clegg A, British Geriatrics Society, et al. Best practice guidelines for the management of frailty: a British geriatrics Society, age UK and Royal College of general practitioners report. Age Ageing 2014;43:744-7.

6 Theou O, Squires E, Mallery K, et al. What do we know about frailty in the acute care setting? A scoping review. BMC Geriatr 2018;18:139.

7 Morley JE, Vellas B, van Kan GA, et al. Frailty consensus: a call to action. J Am Med Dir Assoc 2013;14:392-7.

8 Rodríguez-Mañas L, Féart C, Mann G, et al. Searching for an operational definition of frailty: a Delphi method based consensus statement: the frailty operative definition-consensus conference project. J Gerontol A Biol Sci Med Sci 2013;68:62-7.

9 Dent E, Kowal P, Hoogendijk EO. Frailty measurement in research and clinical practice: a review. Eur J Intern Med 2016;31:3-10.

10 Dent E, Martin FC, Bergman H, et al. Management of frailty: opportunities, challenges, and future directions. Lancet 2019;394:1376-86.

11 Walston J, Bandeen-Roche K, Buta B, et al. Moving frailty toward clinical practice: Nla intramural frailty science symposium summary. J Am Geriatr Soc 2019;67:1559-64.

12 Fried LP, Tangen CM, Walston J, et al. Frailty in older adults: evidence for a phenotype. J Gerontol A Biol Sci Med Sci 2001;56:M146-57.

13 Mitnitski AB, Mogilner AJ, Rockwood K. Accumulation of deficits as a proxy measure of aging. ScientificWorldJournal 2001;1:323-36.

14 Buta BJ, Walston JD, Godino JG, et al. Frailty assessment instruments: systematic characterization of the uses and contexts of highly-cited instruments. Ageing Res Rev 2016;26:53-61.

15 Clegg A, Bates C, Young J, et al. Development and validation of an electronic frailty index using routine primary care electronic health record data. Age Ageing 2016;45:353-60.

16 Cesari M, Franchi C, Cortesi L, et al. Implementation of the frailty index in hospitalized older patients: results from the REPOSI register. Eur J Intern Med 2018;56:11-18.

17 Avila-Funes JA, Amieva H, Barberger-Gateau P, et al. Cognitive impairment improves the predictive validity of the phenotype of frailty for adverse health outcomes: the three-city study. J Am Geriatr Soc 2009;57:453-61.

18 Boyle PA, Buchman AS, Wilson RS, et al. Physical frailty is associated with incident mild cognitive impairment in communitybased older persons. J Am Geriatr Soc 2010;58:248-55.

19 Swiss Personalized Health Network. Swiss personalized health network (SPHN), 2017. Available: https://www.sphn.ch/en/about.html [Accessed 25 Jul 2019].

20 Hope AA, Adeoye O, Chuang EH, et al. Pre-hospital frailty and hospital outcomes in adults with acute respiratory failure requiring mechanical ventilation. J Crit Care 2018;44:212-6.

21 Wan X, Wang W, Liu J, et al. Estimating the sample mean and standard deviation from the sample size, median, range and/or interquartile range. BMC Med Res Methodol 2014;14:135.

22 Evans SJ, Sayers M, Mitnitski A, et al. The risk of adverse outcomes in hospitalized older patients in relation to a frailty index based on a comprehensive geriatric assessment. Age Ageing 2014;43:127-32.

23 Hewitt J, Long S, Carter B, et al. The prevalence of frailty and its association with clinical outcomes in general surgery: a systematic review and meta-analysis. Age Ageing 2018;47:793-800.

24 Hogan DB, Maxwell CJ, Afilalo J, et al. A scoping review of frailty and acute care in middle-aged and older individuals with recommendations for future research. Can Geriatr J 2017;20:22-37.

25 Song X, Mitnitski A, Rockwood K. Prevalence and 10-year outcomes of frailty in older adults in relation to deficit accumulation. $J$ Am Geriatr Soc 2010;58:681-7.

26 Rockwood K, Song X, Mitnitski A. Changes in relative fitness and frailty across the adult lifespan: evidence from the Canadian national population health survey. CMAJ 2011;183:E487-94.

27 Rockwood K, Andrew M, Mitnitski A. A comparison of two approaches to measuring frailty in elderly people. J Gerontol A Biol Sci Med Sci 2007;62:738-43.

28 Gaudet-Blavignac C, Raisaro JL, Touré V, et al. A National, Semantic-Driven, Three-Pillar Strategy to EnableHealth Data Secondary Usage Interoperability for Research Withinthe Swiss Personalized Health Network: Methodological Study. JMIR Med Inform 2021;9:e27591.

29 Meier-Abt PJ, Lawrence AK, Selter L. The Swiss approach to precision medicine. Swiss Med Wkly 2018.

30 Swiss Institute of Bioinformatics. Personalized health informatics group. 2018, 2019. Available: https://www.sib.swiss/research- 
infrastructure/personalized-health-2/personalized-health-informaticsgroup [Accessed 25 Jul 2019].

31 D'Ath P, Katona P, Mullan E, et al. Screening, detection and management of depression in elderly primary care attenders. I: the acceptability and performance of the 15 item geriatric depression scale (GDS15) and the development of short versions. Fam Pract 1994;11:260-6.

32 Shah A, Herbert R, Lewis S, et al. Screening for depression among acutely ill geriatric inpatients with a short geriatric depression scale. Age Ageing 1997;26:217-21.

33 Bischoff-Ferrari HA, de Godoi Rezende Costa Molino C, Rival S, et al. DO-HEALTH: Vitamin D3 - Omega-3 - Home exercise - Healthy aging and longevity trial - Design of a multinational clinical trial on healthy aging among European seniors. Contemp Clin Trials 2021;100:106124.
34 Bischoff-Ferrari HA, Vellas B, Rizzoli R, et al. Effect of vitamin D supplementation, omega-3 fatty acid supplementation, or a Strength-Training exercise program on clinical outcomes in older adults: the DO-HEALTH randomized clinical trial. JAMA 2020;324:1855-68.

35 Gagesch M, Abderhalden L, Kressig RW. Threshold definition for grip strength to identify relevant weakness in Swiss DO-HEALTH participants. International Conference on Frailty and Sarcopenia Research; February 20-22, 2019, Miami Beach, FL, 2019.

36 Börsch-Supan A, Brugiavini A. Health, Ageing and Retirement in Europe - First Results from the Survey of Health, Ageing and Retirement in Europe. Mannheim Research Institute for the Economics of Aging (MEA), 2008. 